\title{
Treatment choice, duration, and cost in patients with interstitial cystitis and painful bladder syndrome
}

\author{
Jennifer T. Anger • Nasim Zabihi • \\ J. Quentin Clemens • Christopher K. Payne • \\ Christopher S. Saigal • Larissa V. Rodriguez
}

Received: 12 June 2010 / Accepted: 4 August 2010/Published online: 2 September 2010

(C) The Author(s) 2010. This article is published with open access at Springerlink.com

\begin{abstract}
Introduction and hypothesis In order to better understand provider treatment patterns for interstitial cystitis (IC)/painful bladder syndrome, we sought to document the therapies utilized and their associated expenditures using a national dataset.

Methods A cohort was created by applying the ICD-9 diagnosis of IC (595.1) to INGENIX claims for the year 1999. Subjects were followed for 5 years, and patterns of care and related expenditures were evaluated.

Results Of 553,910 adults insured in 1999, 89 subjects had a diagnosis of IC with 5 -year follow-up data. All subjects were treated with oral medication(s), $26 \%$ received intravesical treatments, and $22 \%$ underwent hydrodistension. Total expenditures per subject were $\$ 2,808$.

Conclusions The majority of IC expenditures were attributable to oral medical therapy. Hydrodistension and intravesical instillations were utilized in less than $25 \%$ of patients. Hydrodistension was used more frequently among subjects with a new diagnosis; this may reflect its utilization as part of a diagnostic algorithm.
\end{abstract}

Presented at the Society for Urodynamics and Female Urology Annual Meeting, Las Vegas, February 2009

J. T. Anger · N. Zabihi · C. S. Saigal · L. V. Rodriguez $(\bowtie)$

Department of Urology, UCLA,

Box 951738, Los Angeles, CA 90095-1738, USA

e-mail: LRodriguez@mednet.ucla.edu

J. Q. Clemens

Department of Urology, University of Michigan,

Ann Arbor, MI, USA

C. K. Payne

Department of Urology, Stanford University,

Palo Alto, CA, USA
Keywords Interstitial cystitis - Treatment · Bladder pain . Cost $\cdot$ Claims data $\cdot$ Ingenix

\section{Introduction}

Interstitial cystitis/painful bladder syndrome (IC/PBS) is a debilitating disease that presents with a constellation of symptoms including pelvic pain, urinary urgency, frequency, nocturia, and small voided volumes in the absence of other identifiable etiologies. Reports of its prevalence vary; it is reported to affect 10/100,000 of the population in Finland[1]. In 1989 it was estimated to affect 501/100,000 individuals $(0.5 \%)$ in the US [2]. Studies have shown that this disease significantly impacts quality of life; patients with IC/PBS score lower than women without IC/PBS in four out of seven dimensions measured by the short-form health survey (SF-36) questionnaire including physical function, vitality, social function, and bodily pain domains [3]. A study in a population of managed care patients in the US demonstrated that this disease is underreported; the prevalence may be 30-50-fold higher in women and 60 100 -fold higher in men[4].

The economic burden of IC/PBS is significant. Incremental medical costs are estimated to exceed $\$ 100$ million per year [5] and total income lost to IC in 1987 was estimated to be from $\$ 177$ to $\$ 311$ million [6]. In a study by the Urologic Diseases in America Project, the average total annual medical cost per person with an IC/PBS diagnosis was $\$ 7,597$; more than double the figure for those without the diagnosis, after controlling for several factors [7]. A study of a managed care population found costs associated with IC/PBS to be $\$ 4,000$ greater than for age-matched controls [8]. A multimodal treatment approach is usually employed in treating these patients. The goal of this study is to better understand 
provider treatment patterns and likely treatment efficacy through the use of a national dataset.

\section{Methods}

This study was part of the Urologic Diseases in America Project. Ingenix is a claims-based dataset, which captures utilization of medical services for approximately 1.8 million employees, retirees, and dependants of 25-large Fortune 500 employers [9]. The sample used consisted of primary beneficiaries, age 18-64 years, who were continuously enrolled for the year 1999. A cohort was created by applying the ICD-9 diagnosis code for IC/PBS (595.1) to claims for the year 1999. We did not exclude the diagnosis of overactive bladder (OAB), partly because there is no ICD9 code specific for OAB other than 596.51 (hypertonicity of bladder). Since many of the symptoms of OAB overlap with IC codes, such as frequency/urgency/nocturia, and OAB is not easily diagnosed with codes, we chose not to exclude these patients. Although it is possible that there are cases that were misdiagnosed, we suspect that this number is small. This cohort was followed for 5 years in order to obtain longterm data on these subjects. Claims for both prevalent and incident cases were analyzed. Incident cases were identified by excluding subjects with a claim for IC/PBS in 1998 .

The medical claims in the Ingenix dataset include financial information, diagnosis, and procedure codes, drug claims, and national drug codes which were used to examine utilization of specific drugs. We identified oral medications and procedures utilized as well as their duration of use and associated expenditures; Appendix 1 identifies the medications queried and Appendix 2 summarizes procedures and their associated CPT-4 procedure codes.

\section{Results}

In the year 1999, a total of 553,910 individuals were covered. A total of 321 women had IC/PBS in 1999, including both incident and prevalent cases. Eighty-nine women had followup data for 5 years; out of these, 54 subjects were incident cases, i.e., they had no claims for IC/PBS in 1998 and had IC/ PBS claims in 1999. The mean and median age in the total insured population in the dataset was 58.5 and 59 years, respectively. The mean and median age among the cohort with IC/PBS was 63.3 and 65 years, respectively, and that of the incident cases was 63 and 64.5 years. The age distribution of the cohort is shown in Table 1.

All patients had at least one claim for an oral medication indicated for symptoms of IC/PBS. Medications used by more than $10 \%$ of the subjects in prevalent and incident cases, respectively, were PPS $(35 \%, 15 \%)$, tolterodine $(31 \%, 31 \%)$, amitriptyline $(25 \%, 13 \%)$, gabapentin $(19 \%, 15 \%)$, and oxybutinin $(18 \%, 17 \%)$; the average duration of therapy for all of these medications in all patients was 72 weeks. Among medications used to treat IC/PBS, anticholinergics were the most common class utilized $(49 \%$ for tolterodine and oxybutinin combined, Table 2). Narcotic pain medications were utilized by $84 \%$ of the subjects (Table 3 ).

Twenty-two percent of all subjects and $30 \%$ of the incident cases underwent hydrodistension; intravesical therapies were used in treatment of $26 \%$ and $17 \%$ of all and incident cases, respectively (Table 4). These therapies were not repeated when used, with the exception of one patient who had a repeat intravesical instillation. Additionally, when treated with intravesical therapies, not all subjects received a full 6week course of treatment; treatments ranged from 2 to 6 weeks, with an average of 4.4 weeks.

Expenditures for all IC/PBS-specific treatments combined for the cohort were $\$ 2,808$ per patient over 5 years. This does not include related expenditures for physician evaluation, laboratory and/or radiology testing. Oral medical therapy represented $82 \%$, hydrodistension $15 \%$, and intravesical instillations $3 \%$ of the overall expenditures. Among the medications used by more than $10 \%$ of the subjects, PPS was the most costly at $\$ 36 /$ week, followed by gabapentin at $\$ 20 /$ week, oxybutinin at $\$ 18 /$ week, and tolterodine at $\$ 17 /$ week. The duration of utilization was the highest for PPS (99.5 weeks), followed by gabapentin ( 87.4 weeks), oxybutinin (55.3 weeks) and tolterodine (48.5 weeks).

\section{Discussion}

This study has several important findings that shed light on the patterns of care for adults with IC/PBS. First, we found that all of the subjects were treated with at least one oral
Table 1 Age distribution of patients with IC in cohort (prevalent and incident cases)

\begin{tabular}{llclc}
\hline Age (years) & Number & Percent (\%) & Cumulative frequency & Cumulative percent (\%) \\
\hline $21-30$ & 3 & 3.37 & 3 & 3.37 \\
$31-40$ & 4 & 4.49 & 7 & 7.87 \\
$41-50$ & 11 & 12.36 & 18 & 20.22 \\
$51-65$ & 29 & 32.58 & 47 & 52.81 \\
$>65$ & 42 & 47.19 & 89 & 100.00 \\
\hline
\end{tabular}


Table 2 Utilization and cost of oral medication for IC

\begin{tabular}{llccrr}
\hline & Drug & $N$ treated & Percentage treated (\%) & Duration (weeks) & Total Cost \\
\hline \multirow{2}{*}{ All cases } & Detrol & 28 & 31 & 48.52 & $\$ 23,265$ \\
& Ditropan & 16 & 18 & 55.30 & $\$ 16,123$ \\
& Neurontin & 17 & 19 & 87.43 & $\$ 30,332$ \\
& Elmiron & 31 & 35 & 99.55 & $\$ 112,144$ \\
& Elavil & 22 & 25 & 67.86 & $\$ 2,375$ \\
\multirow{5}{*}{ Incident cases } & Detrol & 17 & 31 & 43.14 & $\$ 11,478$ \\
& Ditropan & 9 & 17 & 64.49 & $\$ 9,290$ \\
& Neurontin & 8 & 15 & 77.93 & $\$ 13,660$ \\
& Elmiron & 8 & 15 & 81.61 & $\$ 26,120$ \\
& Elavil & 7 & 13 & 32.63 & $\$ 365$ \\
\hline
\end{tabular}

medication used to treat patients with IC/PBS during the study period. Of these, narcotics were the most commonly utilized class of medications. This might indicate that many commonly prescribed IC/PBS-specific medications are less effective than narcotics. Alternatively, the narcotics may have been used to treat other pain complaints arising from sites other than the bladder. Anticholinergics were the second most commonly utilized class of medications used by $49 \%$ of subjects (tolterodine and oxybutinin combined) with an average utilization period of approximately 1 year. This utilization period is actually longer than that documented for women with overactive bladder symptoms $[10,11]$. In the Interstitial Cystitis Data Base (ICDB) study, a multicenter, observational study designed to document the treatment history of IC/PBS and patient characteristics, only $2-4 \%$ of patients received anticholinergics as monotherapy or in combination with other medications [12]. However, this was a select cohort of patients who were recruited from a limited number of tertiary care centers. Our data suggest that anticholinergic agents are used much more widely in the community setting. This observation reinforces the inherent difficulty in distinguishing between the clinical syndromes of overactive bladder and IC/PBS, since many of symptoms overlap. It is possible that there is therapeutic benefit gained from this group of medications by patients with IC/PBS, or alternatively, they may have seen a slight benefit with little side effects and are willing to continue these medications since they were not offered other therapies.

A substantial minority of patients were treated with centrally acting medications including gabapentin and tricyclic antidepressants. These medications have been used in treatment of chronic pain conditions such as Complex Regional Pain Syndrome Type-I (CPRS-I) with success. It has been suggested that the pathophysiology of IC/PBS may partly be due to deregulation of the central nervous system, similar to CPRS-I [13]. Gabapentin, an antiepileptic, has been effective in treatment of chronic sympathetically mediated pain syndromes and there are reports of its efficacy in treatment of IC/PBS [13] [14]. Tricylic antidepressants such as amitriptyline also have demonstrated efficacy in treating patients with IC/PBS [15-17]. In our cohort, gabapentin and amitriptyline were among the five most commonly used medications and were used to treat $19 \%$ and $25 \%$ of patients, respectively. This is slightly higher than the $17 \%$ of subjects treated with amitriptyline in the ICDB study [15-17]. Given the theory that the etiology of IC/PBS/chronic pelvic pain may reside partly in the central nervous system, a shift toward treatments aimed at regulating the nervous system is logical. While claims data cannot be used to infer the reasons for the low rate of utilization of centrally acting medications in the Ingenix cohort, possible explanations include a lack of provider awareness of the potential role of central nervous

Table 3 Narcotic usage among patients with IC

\begin{tabular}{lrrr}
\hline & $\begin{array}{l}\text { IC in 1999 } \\
(N=89)\end{array}$ & $\begin{array}{l}\text { IC in 1999, no IC in 1998 } \\
(N=54)\end{array}$ & $\begin{array}{l}\text { IC in 1999 and 1998 } \\
(N=35)\end{array}$ \\
\hline Number of people who took any narcotic in 1999 & 40 & 22 \\
Percentage of people who took any narcotic in 1999 (\%) & 45 & 41 & 51 \\
Total number of scripts filled in 1999 & 90 & 40 & 50 \\
Total day supply of narcotics in 1999 & 1,302 & 485 & 45 \\
Number of people who took any narcotic 1999-2003 & 75 & 83 & 30 \\
Percentage people took any narcotic 1999-2003 (\%) & 84 & 250 & 36 \\
Total number of scripts filled 1999-2003 & 623 & 4,731 & 6,466 \\
Total day supply of narcotics 1999-2003 & 11,197 & 373
\end{tabular}


Table 4 Utilization and cost of intravesical treatment and hydrodistention

\begin{tabular}{lllll}
\hline & Treatment & $N$ treated & Percentage treated (\%) & Total cost \\
\hline \multirow{2}{*}{ All cases } & Intravesical & 23 & 26 & $\$ 8,022$ \\
& Hydrodistension & 20 & 22 & $\$ 37,676$ \\
\multirow{2}{*}{ Incident cases } & Intravesical & 9 & 17 & $\$ 3,648$ \\
& Hydrodistension & 16 & 30 & $\$ 30,141$ \\
\hline
\end{tabular}

system dysregulation in this syndrome or low perceived efficacy of available agents to mitigate this dysregulation. Further prospective work in this area would be helpful to further interpret this finding.

In our cohort, PPS was utilized by $35 \%$ of all subjects and $15 \%$ of the incident cases with a mean utilization period of 99.5 and 82 weeks, respectively. PPS is the only oral medication approved by the Food and Drug Administration (FDA) for the treatment of IC/PBS. One theory of the pathophysiology of IC/PBS implicates a defect in the bladder glycosaminoglycan layer as partially responsible for symptoms of IC/PBS. PPS, which is available in oral formulation and is excreted in urine, is prescribed with the intent to correct this defect [18]. The studies evaluating its efficacy have shown a wide array of clinical responses. A multicenter randomized controlled trial showed that $32 \%$ of those on PPS compared to $16 \%$ of patients on placebo reported more than $50 \%$ improvement in a global self-evaluation of their symptoms [18]. However, the Interstitial Cystitis Clinical Trials Group conducted a placebo-controlled trial to evaluate the efficacy of PPS and hydroxyzine, and found no statistically significant benefit to treatment with PPS.[19] In a systematic review of the pharmacologic management of IC/ PBS by Dimitrakov et al., the pooled estimate of the effect of pentosan polysulfate therapy suggested a modest benefit, with a relative risk of 1.78 for patient-reported improvement in symptoms (95\% confidence interval, 1.34-2.35)[20]. The long utilization period identified in our database also points to at least some therapeutic benefit enjoyed by the subjects. Alternatively, it may be a result of the manufacturer's recommendations that long-term therapy (greater than 1 year) may be needed before a clinical effect occurs.

We also found that more patients who were newly diagnosed with IC/PBS (31\%) underwent hydrodistension, compared to those with a previous diagnosis $(11 \%)$, and that the procedure was not repeated at a high rate. Hydrodistension has historically been used both for diagnosis and treatment of IC/PBS; however, after the NIH Interstitial Cystitis Database study documented that over $60 \%$ of patients regarded as having IC would have been excluded if the NIDDK criteria were applied[21], the diagnostic value of hydrodistension in clinical practice has been questioned[22]. The ICDB study similarly revealed that hydrodistension was utilized more frequently among those newly diagnosed (48.4\%) compared to those with a previous diagnosis $(25.7 \%)$ [16]. Our findings could partly reflect the use of this procedure as a diagnostic tool more frequently and less so as a therapeutic maneuver. It is also possible that when used for therapy, it was not repeated due to poor efficacy.

In our cohort, $26 \%$ of the patients had intravesical instillations; the average number of instillations ranged from two to six. A variety of Intravesical instillations have been used to treat IC/PBS, including silver nitrate, marcaine/ lidocaine, dimethyl sulfoxide (DMSO), hyaluronic acid, heparin, PPS, Bacillus Calmette-Guerin (BCG), and rosiniferatoxin. DMSO is the only FDA approved intravesical agent for the treatment of this condition. The available Ingenix data do not allow us to identify the exact agent used for the instillations. These instillations are sometimes given as a 4 6 week course of therapy, while at other times they are given as 'rescue' therapy for symptom flares. These different uses may explain the variable numbers of instillations observed in our dataset.

A summation of all individual incremental care in patients with IC/PBS, exclusive of indirect costs, is estimated at $\$ 100$ million in the US alone[5], and medical expenditures among IC/PBS patients are double the figure associated with those without the disease[23]. In our cohort, the overall expenditures related to the treatment of IC/PBS were $\$ 2,808$ per subject over 5 years, the majority of which were related to oral medical therapy. PPS, the only FDA approved medication, was the most expensive at $\$ 36$ per week. The weekly cost of $\$ 18$ for oxybutynin and tolterodine is likely related to the use of the brand name or extended release formulations, which are more costly than short-acting formulas.

Although detailed clinical information cannot be obtained from claims-based data, claims data provides information about real-world practice patterns, including pharmacy care, in a large population of individuals. However, this study, like may claims-based analyses, has limitations. It is also important to note that this project is not designed to study the epidemiology of the disease, or to understand the rationale behind treatment, but rather studies a cohort of patients from 1 year treated by multiple providers. Claims-based data are designed for billing purposes, and therefore lack important information about severity of illness and reasons for treatment discontinuation. Coding is often incomplete or inaccurate, and our cohort may have included some patients with overactive bladder symptoms or other types of pelvic pain unrelated to IC/PBS. It is also possible that we excluded some subjects if their condition was coded using a combination of ICD-9 codes such as bladder pain and urgency/ 
frequency, since we identified the cohort by the ICD-9 diagnosis for IC/PBS (595.1). Also, our cohort was older (mean age 63.3 years) than the reported mean age for patients with IC/PBS (age range 43-59 years) [2, 19,24,25]. This could be partly due to the population under study; the population studied consists of the retirees, employees, and their dependants with a mean and median age of 58.5 and 59 years, respectively during the study year of 1999. Thus, our findings may not be entirely generalizable to a younger IC/PBS population.

\section{Conclusions}

The majority of treatment costs for IC/PBS were attributable to oral medical therapy. Anticholinergics, PPS, tricyclic antidepressants, and gabapentin are the most commonly used group of such treatments. Subjects likely tolerated these medications well, as the average length of treatment exceeded 1 year. Hydrodistension and intravesical instillations were utilized in less than a quarter of the patients and not repeated when used. This may be due to its utilization as part of diagnostic algorithm more frequently than as a treatment modality.

Acknowledgments This work was funded by the NIDDK as part of the Urologic Diseases in America Project.

Conflicts of interest JQ Clemens: Merck, Investment interest; Pfizer, consultant; Lilly, consultant; Medtronics, proctor. Payne C: Allergan, consultant; Astellas, consultant; Celgene, Investigator; Coloplast, Investigator; Curant, Investment Interest; Medtronic, Investigator. All other authors have no conflict of interest.

Open Access This article is distributed under the terms of the Creative Commons Attribution Noncommercial License which permits any noncommercial use, distribution, and reproduction in any medium, provided the original author(s) and source are credited.

\section{Appendix 1}

Table 5 List of oral medications queried

\begin{tabular}{ll}
\hline Oral medical therapy & \\
\hline Tricyclic antidepressants & Elavil (amitriptyline) \\
& Norpramin (desipramine) \\
& Pamelor (nortriptyline) \\
& Sinequan (doxepin) \\
& Tofranil (imipramine) \\
& Vistaril (hydroxyzine pamoate) \\
Antihistamines & Atarax (hydroxyzine hydrochloride) \\
& Tagamet (cimetidine) \\
H2 blockers & Zantac (ranitidine)
\end{tabular}

Table 5 (continued)

Oral medical therapy

\begin{tabular}{ll}
\hline Leukotriene inhibitors & Singulair (montelukast) \\
& Accolate (zafirlukast) \\
Anticonvulsants & Neurontin (gabapentin) \\
& Klonopin (clonazepam) \\
Antispasmotic and analgesic & Urimax \\
& Pyridium \\
& Pyridium-plus \\
& Urised \\
& Ditropan, Ditropan XL (oxybutinin) \\
Antispasmotic & Detrol, Detrol LA (tolterodine) \\
andanticholinergic & Levsin, Levbid, Levsinex \\
& (hyoscyamine) \\
& Urispas (Flavoxate) \\
& Bentyl (dicyclomine) \\
& Pro-Banthine (propantheline) \\
Mucosal surface protectants & Elmiron
\end{tabular}

\section{Appendix 2}

Table 6 IC treatments and corresponding CPT codes

\begin{tabular}{|c|c|c|}
\hline \multirow[t]{10}{*}{$\begin{array}{l}\text { Treatment } \\
\text { Intravesical }\end{array}$} & Procedure & $\begin{array}{l}\text { CPT code } \\
51700,51720\end{array}$ \\
\hline & Marcaine & $\mathrm{J} 3490$ \\
\hline & Lidocaine & $\mathrm{J} 2000$ \\
\hline & Heparin & $\mathrm{J} 1644$ \\
\hline & $\begin{array}{l}\text { Dimethyl sulfoxide } \\
\text { (DMSO) } \\
\text { Hyaluronic acid }\end{array}$ & $\mathrm{J} 1212$ \\
\hline & Chondroitin sulfate & 82485 \\
\hline & $\begin{array}{l}\text { Pentosan } \\
\text { polysulfate }\end{array}$ & \\
\hline & $\mathrm{BCG}$ & 90586, J9031 \\
\hline & Resiniferatoxina & \\
\hline & Botox & J0585 \\
\hline \multirow{2}{*}{$\begin{array}{l}\text { Cystoscopy } \\
\text { under anesthesia }\end{array}$} & & 52000 and 00910 \\
\hline & Hydrodistension & 52260 \\
\hline \multirow[t]{6}{*}{ Surgical } & $\begin{array}{l}\text { Interstim } \\
\text { (neuromodulation) }\end{array}$ & $\begin{array}{r}64681,64590,64555 \\
64561,64575,64581\end{array}$ \\
\hline & $\begin{array}{l}\text { Removal/revision } \\
\text { Interstim }\end{array}$ & 64585,64595 \\
\hline & Cystectomy & $\begin{array}{r}51550,51555,51565, \\
51590,51595,51596\end{array}$ \\
\hline & $\begin{array}{l}\text { Augmentation } \\
\text { cystoplasty }\end{array}$ & 51960 \\
\hline & Urinary diversion & $51596,51595,51590$ \\
\hline & Neobladder & 51596 \\
\hline
\end{tabular}




\section{References}

1. Oravisto (1975) Epidemiology of interstitial cystitis. Ann Chir Gynaecol Fenn 64:75-77

2. Jones CA, Nyberg L (1997) Epidemiology of interstitial cystitis. Urology 49:2-9

3. Michael YL, Kawachi I, Stampfer MJ, Colditz GA, Curhan GC (2000) Quality of life among women with interstitial cystitis. J Urol 164:423-427

4. Clemens JQ, Meenan RT, O'Keeffe Rosetti MC, Brown SO, Gao SY, Calhoun EA (2005) Prevalence of interstitial cystitis symptoms in a managed care population. J Urol 174:576-580

5. Nickel JC (2004) Interstitial cystitis: a chronic pelvic pain syndrome. Med Clin North Am 88:467-481

6. Sant GR (1997) Interstitial cystitis. Lippincott-Raven, Philadelphia

7. Clemens JQ, Joyce GF, Wise M, Payne CK (2004) Interstitial cystitis and painful bladder syndrome. In: Litwin MS, Saigal CS (eds) Urologic diseases in America. US Department of Health and Human Services, Public Health Service, National Institutes of Health, National Institute of Diabetes and Digestive and Kidney Diseases. US Government Publishing Office, Washington, pp 283-316, NIH Publication No. 04-5512

8. MR CJQ, Rosetti MC, Kimes T, Calhoun EA (2008) Costs of interstitial cystitis in a managed care population. Urology 71:776-780

9. Saigal CS, Joyce GF, Geschwind SA, Litwin MS (2004) Methods. In: Litwin MS, Saigal CS (eds) Urologic Diseases in America. US Department of Health and Human Services, Public Health Service, National Institutes of Health, National Institute of Diabetes and Digestive and Kidney Diseases. US Government Publishing Office, Washington, pp 283-316, NIH Publication No. 04-5512

10. Dmochowski R (2005) Improving the tolerability of anticholinergic agents in the treatment of overactive bladder. Drug Saf 28:583-600

11. Dmochowski RR, Starkman JS, Davila GW (2006) Transdermal drug delivery treatment for overactive bladder. Int Braz J Urol 32:513-520

12. Simon LJ, Landis JR, Erickson DR, Nyberg LM (1997) The interstitial cystitis data base study: concepts and preliminary baseline descriptive statistics. Urology 49:64-75
13. Janicki TI (2003) Chronic pelvic pain as a form of complex regional pain syndrome. Clin Obstet Gynecol 46:797-803

14. Hansen HC (2000) Interstitial cystitis and the potential role of gabapentin. S Med J 93:238-242

15. van Ophoven A, Hertle L (2005) Long-term results of amitriptyline treatment for interstitial cystitis. J Urol 174:1837-1840

16. Rovner E, Propert KJ, Brensinger C, Wein AJ, Foy M, Kirkemo A, Landis JR, Kusek JW, Nyberg LM (2000) Treatments used in women with interstitial cystitis: the interstitial cystitis data base (ICDB) study experience. The interstitial cystitis data base study group. Urology 56:940-945

17. van Ophoven A, Pokupic S, Heinecke A, Hertle L (2004) A prospective, randomized, placebo controlled, double-blind study of amitriptyline for the treatment of interstitial cystitis. J Urol $172: 533-536$

18. Hanno P (2005) Painful bladder syndrome (including interstitial cystitis). In: Abrams P (ed) Incontinence management, vol 2. Health Publication Ltd, Paris, pp 130-137

19. Sant GR, Propert KJ, Hanno PM, Burks D, Culkin D, Diokno AC et al (2003) A pilot clinical trial of oral pentosan polysulfate and oral hydroxyzine in patients with interstitial cystitis. J Urol 170:810-815

20. Dimitrakov J, Kroenke K, Steers WD, Berde C, Zurakowski D, Freeman MR et al (2007) Pharmacologic management of painful bladder syndrome/interstitial cystitis: a systematic review. Arch Intern Med 167:1922-1929

21. Hanno PM, Landis JR, Matthews-Cook Y, Kusek J, Nyberg L Jr (1999) The diagnosis of interstitial cystitis revisited: lessons learned from the National Institutes of Health Interstitial Cystitis Database study. J Urol 161:553-557

22. Ottem DP, Teichman JM (2005) What is the value of cystoscopy with hydrodistension for interstitial cystitis? Urology 66:494-499

23. CJ PCK, Joyce G, Pace J (2005) Medical cost of interstitial cystitis and painful bladder syndrome. J Urol 173:83

24. Nickel JC, Teichman JM, Gregoire M, Clark J, Downey J (2005) Prevalence, diagnosis, characterization, and treatment of prostatitis, interstitial cystitis, and epididymitis in outpatient urological practice: the Canadian PIE Study. Urology 66:935-940

25. Hanno PM (1990) Interstitial cystitis. Springer-Verlag, London 